\title{
Hedgehog-inspired magnetic nanoparticles for effectively capturing and detecting exosomes
}

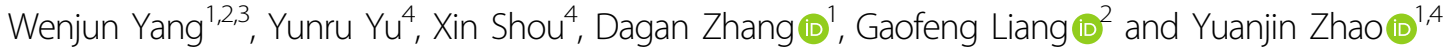

\begin{abstract}
Exosomes, as one type of extracellular vesicle derived from multiple cells, have much potential as cancer biomarkers in clinical applications, but their enrichment and detection remain a huge challenge. Herein, inspired by the burr-like structure of the hedgehog, we present a new nanoparticle with a nanoneedle-assembled shell and a magnetic core for the effective capture and detection of exosomes. The unique nanoneedle structures endowed the magnetic nanoparticles with a large surface area for antibody modification so that the nanoparticles could serve as a platform for efficient exosome capture. In addition, the controllable movement of exosome-combined nanoparticles, which is due to the magnetic iron oxide cores, provides tremendous convenience for separating exosomes. The practical value of these nanoparticles in exosome analyses of serum from healthy patients and patients with liver cancer has also been demonstrated. Thus, we believe that bioinspired hierarchical nanoparticles are promising for biomedical and clinical applications.
\end{abstract}

\section{Introduction}

Exosomes are one type of extracellular vesicle important in intercellular communication ${ }^{1,2}$. As vesicles of endosomal origin secreted from cells, exosomes have multiple functional components, such as nucleic acids, proteins, and metabolites ${ }^{3}$. A correlation between tumor-derived exosomes and tumor pathogenesis and progression has been observed ${ }^{4-7}$. Therefore, tumor-derived exosomes have been considered a novel cancer biomarker that could be used for tumor diagnosis, treatment, and prognosis ${ }^{8-13}$. Although many detection methods have been developed for exosome biomarkers, the insufficient concentration of isolated exosomes limits their efficiency and preci$\operatorname{sion}^{14,15}$. Thus, exosome enrichment and purification are of great importance for subsequent analysis. To fulfill this,

\footnotetext{
Correspondence: Dagan Zhang (zhang_dagan@126.com) or

Gaofeng Liang (lgfeng990448@163.com) or Yuanjin Zhao (yjzhao@seu.edu.cn)

'Department of Clinical Laboratory, Institute of Translational Medicine, The

Affiliated Drum Tower Hospital of Nanjing University Medical School, Nanjing,

China

${ }^{2}$ School of Basic Medical Sciences, Henan University of Science and

Technology, Luoyang, China

Full list of author information is available at the end of the article

These authors contributed equally: Wenjun Yang, Yunru Yu
}

different approaches have been proposed, including ultracentrifugation, sucrose density gradient centrifugation, and immune-magnetic beads ${ }^{16}$. Among these methods, exosome enrichment based on magnetic beads has attracted increasing attention ${ }^{17-20}$. Although significant progress has been made, the method still has some nonnegligible shortcomings that limit its applications; for example, conventional magnetic beads often have simple surface structures, few immobilized probes, and inefficient separation even with complex surface modification $^{21,22}$. Thus, a convenient and effective strategy for tumor-derived exosome enrichment and identification is still needed in clinical research.

In this paper, inspired by the fascinating burr-like structure of hedgehog, we present novel magnetic particles with nanoneedles on their surface for capturing and detecting exosomes effectively, as shown in Fig. 1. The burr-like structure not only provides excellent structural stability but also brings about extraordinary surface functions, which enables creatures to perform tough tasks, such as protein purification, cell separation, and enzyme immobilization $^{23-28}$. Because the features of these structural-functional adaptations have inspired scientists, 


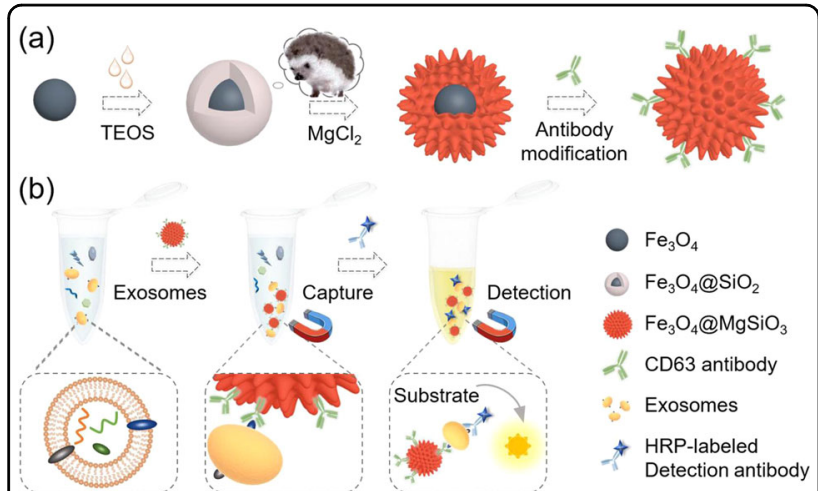

Fig. 1 Schematic illustration. a Schematic illustration of the preparation and modification process of the $\mathrm{Fe}_{3} \mathrm{O}_{4} @ \mathrm{MgSiO}_{3}$ nanoparticles. b Schematic illustration of isolating and detecting exosomes with $\mathrm{Fe}_{3} \mathrm{O}_{4} @ \mathrm{MgSiO}_{3}$ nanoparticles.

extensive research has been performed on the preparation of hedgehog-like materials ${ }^{29-32}$. Compared to nanoparticles made through other fabrication methods, silicate hollow nanoparticles with hedgehog-like structures have been reported to show superior characteristics in that they have a larger surface area and great biocompatibility ${ }^{33-36}$. However, the derived hollow nanoparticles often have a simple microstructure and function that cannot be manipulated controllably. In addition, the potential value of these materials in the enrichment and assay of exosomes and related analytical and biomedical applications has not yet been studied ${ }^{37-41}$. Thus, the generation of such burr-structured materials with controllable motions during application is highly anticipated.

Herein, we proposed a new strategy of formulating novel nanoparticles with nanoneedle surface structures for controlled capture and detection of exosomes (Fig. 1). Nanoparticles composed of magnetic iron cores and graded magnesium silicate shells $\left(\mathrm{Fe}_{3} \mathrm{O}_{4} @ \mathrm{MgSiO}_{3}\right)$ were generated via a tailorable coating process. This coating process could form controlled nanoneedle structures on the surface of magnetic nanoparticles without any impact. Benefitting from the hedgehog-like shell, the $\mathrm{Fe}_{3} \mathrm{O}_{4} @ \mathrm{MgSiO}_{3}$ nanoparticles have abundant surfaces to immobilize antibodies so that many exosomes can be efficiently captured. In addition, the magnetic cores endowed the nanoparticles with a rapid magnetic response under external magnetic fields, which is convenient for separating the enriched exosomes. The practical value of these nanoparticles for analyses of exosomes from the serum of healthy patients and those with liver cancer has also been explored. All of the features described indicate that these hedgehog-inspired magnetic nanoparticles are promising candidates for efficient detection and analysis in biomedical and clinical areas.

\section{Experimental}

\section{Materials}

Anhydrous ferric chloride, NaAc, magnesium chloride hexahydrate $\left(\mathrm{MgCl}_{2} 6 \mathrm{H}_{2} \mathrm{O}\right)$, (3-aminopropyl)-triethoxysilane (APTES), tetraethylorthosilicate (TEOS, 98\%), poly(4-styrenesulfonic acid-co-maleic acid) sodium salt (PSSMA), and ammonium hydroxide $\left(\mathrm{NH}_{3} \cdot \mathrm{H}_{2} \mathrm{O}, 28 \%\right)$ were obtained from Aladdin Reagents (China). Ethylene glycol (EG) was pretreated with a sufficient molecular sieve (type 3). Succinic anhydride (SA), 1-ethyl-3-(3dimethylaminopropyl) carbodiimide (EDC), and $N$ hydroxysuccinimide (NHS) were purchased from SigmaAldrich (USA). 2-Morpholinoethanesulfonic acid (MES) was obtained from Solon (USA). Anti-CD63 antibody (ab59479) and fluorescein isothiocyanate (ab18235)labeled anti-CD63 antibody were purchased from Abcam (USA). BSA was purchased from Roche (USA). The human CEA ELISA Kit and human GPC-3 ELISA Kit were purchased from Jianglai Biotechnology (China). The water employed in all experiments was deionized and ultrafiltered by a Millipore Milli-Q system with minimum resistivity of $18 \mathrm{M} \Omega \cdot \mathrm{cm}$.

\section{Synthesis of $\mathrm{Fe}_{3} \mathrm{O}_{4}$ nanoparticles}

$\mathrm{Fe}_{3} \mathrm{O}_{4}$ nanoparticles were synthesized via a "one-pot" hydrothermal method. First, $1.6 \mathrm{mmol}$ of $\mathrm{FeCl}_{3}(0.26 \mathrm{~g})$, $8.8 \mathrm{mmol}$ of NaAc $(1.2 \mathrm{~g}), 1.8 \mathrm{mmol}$ of PSSMA $(0.4 \mathrm{~g})$, and a moderate amount of water $(20-100 \mu \mathrm{L})$ were added to $16 \mathrm{~mL}$ EG pretreated by a molecular sieve. The mixture was stirred for $\sim 30 \mathrm{~min}$, and the process continued after $6 \mathrm{mmol}$ of solid $\mathrm{NaOH}(0.24 \mathrm{~g})$ was added. The resultant mixture was transferred to a $20 \mathrm{~mL}$ autoclave to heat in a muffle furnace for $9 \mathrm{~h}$ at $190^{\circ} \mathrm{C}$.

\section{Synthesis of $\mathrm{Fe}_{3} \mathrm{O}_{4} @ \mathrm{SiO}_{2}$ nanoparticles}

The synthesized magnetic $\mathrm{Fe}_{3} \mathrm{O}_{4} @ P S S M A$ particles were collected with a magnet, washed three times with ethanol, and dispersed in $12 \mathrm{~mL}$ water. Then, $40 \mathrm{~mL}$ ethanol and $2 \mathrm{~mL}$ ammonium hydroxide were added to $6 \mathrm{~mL}$ of the $\mathrm{Fe}_{3} \mathrm{O}_{4} @ P S S M A$ and mixed well. The mixture was sonicated for $\sim 10 \mathrm{~min}$ and then transferred to a $100 \mathrm{~mL}$ three-neck flask while stirring at $6000 \mathrm{rpm}$ in a water bath at $50{ }^{\circ} \mathrm{C}$. After the mixture was stirred vigorously for $10 \mathrm{~min}, 200 \mu \mathrm{L}$ of TEOS was added dropwise to the brown mixture. The prepared $\mathrm{Fe}_{3} \mathrm{O}_{4} @ \mathrm{SiO}_{2}$ nanoparticles were washed with ethanol and water after stirring for an hour and stored with $2 \mathrm{~mL}$ of ethanol for further use.

\section{Preparation of $\mathrm{Fe}_{3} \mathrm{O}_{4} @ \mathrm{MgSiO}_{3}$}

First, $0.25 \mathrm{mmol}$ of $\mathrm{MgCl}_{2} \cdot 6 \mathrm{H}_{2} \mathrm{O}$ and $3.3 \mathrm{mmol}$ of $\mathrm{NH}_{4} \mathrm{Cl}$ were added to $10 \mathrm{~mL}$ of an aqueous solution, and ammonia solution $(500 \mu \mathrm{L}, 28 \%)$ was added to the $\mathrm{Fe}_{3} \mathrm{O}_{4} @ \mathrm{SiO}_{2}$ dispersion prepared above. The mixture was 
then transferred to an autoclave, heated to $140{ }^{\circ} \mathrm{C}$, and maintained for $\sim 14 \mathrm{~h}$. The brown precipitate was collected with a magnet, washed three times, and dispersed in moderate ethanol for further applications.

\section{Characterization}

The geometry and size of the $\mathrm{Fe}_{3} \mathrm{O}_{4} @ \mathrm{MgSiO}_{3}$ nanoparticles were observed by scanning electron microscopy (SEM, Hitachi, SU8010, Japan). The nanostructures of the $\mathrm{Fe}_{3} \mathrm{O}_{4} @ \mathrm{MgSiO}_{3}$ nanoparticles were observed through transmission electron microscopy (TEM, FEI, Talos, USA). The distribution of particle size and surface charges was measured on a Zetasizer Nano ZS (Malvern, ZEN3600, U.K). The phase composition of the $\mathrm{Fe}_{3} \mathrm{O}_{4} @ \mathrm{MgSiO}_{3}$ nanoparticles was measured by X-ray diffraction (XRD, Bruker, D8 Advance, Germany). To compare them to those of the $\mathrm{Fe}_{3} \mathrm{O}_{4} @ \mathrm{SiO}_{2}$ nanoparticles, the BET surface area and pore size distribution of the $\mathrm{Fe}_{3} \mathrm{O}_{4} @ \mathrm{MgSiO}_{3}$ nanoparticles were evaluated through $\mathrm{N}_{2}$ adsorption-desorption measurements at $77 \mathrm{~K}$ (Micromeritics 3Flex, USA). The magnetic properties were determined by a vibrating sample magnetometer (VSM, PPMS DynaCool, USA).

\section{Antibody modification}

The CD63 antibody was immobilized on the surface of the $\mathrm{Fe}_{3} \mathrm{O}_{4} @ \mathrm{MgSiO}_{3}$ nanoparticles through covalent modification. The $\mathrm{NaOH}, 2 \%$ APTES-ethanol solution, and $10 \%$ succinic anhydride (SA) solution treatment of $\mathrm{Fe}_{3} \mathrm{O}_{4} @ \mathrm{MgSiO}_{3}$ nanoparticles modified them with amino and carboxylate groups. Then, the combination of the $\mathrm{Fe}_{3} \mathrm{O}_{4} @ \mathrm{MgSiO}_{3}$ nanoparticles and $\mathrm{CD} 63$ antibody was completed after $3 \mathrm{~h}$ of incubation at $37^{\circ} \mathrm{C}$ in EDC/NHS solution. Finally, the CD63 antibody-modified nanoparticles were obtained after being washed three times with buffer solution and blocked with $1 \%$ BSA. The FITClabeled anti-CD63 antibody (ab18235) served to verify the modification with a concentration range of $0.1-50 \mu \mathrm{g} / \mathrm{mL}$. After incubation with the $\mathrm{Fe}_{3} \mathrm{O}_{4} @ \mathrm{MgSiO}_{3}$ nanoparticles and washing three times, they were observed with optical and fluorescence microscopy. To avoid interfering with the detection of fluorescence of the exosomes, we subsequently used the CD63 antibody (ab59479) to modify the nanoparticles in a concentration range from 0.001 to $10 \mu \mathrm{g} \mathrm{mL}^{-1}$.

\section{Preparation of exosome standards}

HepG2 cells were cultivated in DMEM containing 10\% FBS and $1 \%$ penicillin-streptomycin in an incubator maintained at $5 \% \mathrm{CO}_{2}$ and $37^{\circ} \mathrm{C}$ and then cultivated in DMEM containing exosomal-free FBS for $48 \mathrm{~h}$. Then, the supernatant of the culture was centrifuged at $300 \times g$ and $2000 \times g$ for $10 \mathrm{~min}$. After filtration through a $0.22 \mu \mathrm{m}$ filter, the supernatant of the culture was separated by centrifugation at $120,000 \times g(70 \mathrm{~min} \times 2)$ at $4{ }^{\circ} \mathrm{C}$. The precipitated exosome pellets were resuspended in $200 \mu \mathrm{L}$ PBS buffer and stored at $-80^{\circ} \mathrm{C}$ to form exosome standards. The average nanoparticle tracking analysis (NTA) value of the prepared exosome standards was measured to be $7.4 \pm 0.38 \times 10^{9}$ particles $\mathrm{mL}^{-1}$, and their morphology was observed by TEM.

\section{Enrichment and isolation of exosomes using $\mathrm{Fe}_{3} \mathrm{O}_{4} @ \mathrm{MgSiO}_{3}$ nanoparticles}

After using a $1.5 \mu \mathrm{g}$ CD63 antibody to modify $3 \mathrm{mg}$ $\mathrm{Fe}_{3} \mathrm{O}_{4} @ \mathrm{MgSiO}_{3}$ nanoparticles, the modified nanoparticles were coincubated with $50 \mu \mathrm{L}$ exosomes at $37^{\circ} \mathrm{C}$ on a shaker. During this process, the CD63 antibody modified on the nanoparticles became bound to the specific proteins on the exosomes. Then, they could be isolated from the solution after they were combined by applying a magnetic field for $10 \mathrm{~s}$. The $\mathrm{Fe}_{3} \mathrm{O}_{4} @ \mathrm{MgSiO}_{3}$ nanoparticles with captured exosomes were washed with PBS three times, and the membrane dye PKH26 with a concentration of PKH26 of $2 \times 10^{-6}$ was used to stain the exosomes to indicate their capture. The fluorescence intensity was detected with a microplate reader at an excitation wavelength of $551 \mathrm{~nm}$.

To optimize the exosome capture conditions, the number of exosomes before and after capture was measured with $\mathrm{BCA}$ to quantitatively calculate the capture efficiency. The $\mathrm{Fe}_{3} \mathrm{O}_{4} @ \mathrm{MgSiO}_{3}$ nanoparticles were modified with CD63 antibodies at different concentrations $\left(0.1,1,10,100\right.$, and $\left.1000 \mu \mathrm{g} \mathrm{mL}^{-1}\right)$, and then the $\mathrm{Fe}_{3} \mathrm{O}_{4} @ \mathrm{MgSiO}_{3}$ nanoparticles were incubated with identical concentrations of exosomes under the same capture time (30 min).

\section{Western blot analysis of exosomes}

Western blotting was applied to verify the specific marker protein CD63 in exosomes captured on nanoparticles and the standards, which were pretreated with RIPA lysis buffer on ice for $15 \mathrm{~min}$. The loading buffer was mixed and heated for $5 \mathrm{~min}$ at $100^{\circ} \mathrm{C}$. Then, sodium dodecyl sulfate-polyacrylamide gel electrophoresis (SDS-PAGE) was carried out. After transmembrane incubation, the membrane was incubated overnight at $4{ }^{\circ} \mathrm{C}$ with a primary antibody $(1: 1000)$ against CD63 and then incubated with a secondary antibody for one hour. The image was obtained by a gel image system.

\section{Detection for captured exosomes}

An HRP-assisted immunosorbent assay was employed to detect the protein biomarkers of exosomes. In this process, $100 \mu \mathrm{L}$ HRP-CEA or HRP-GPC was incubated with nanoparticles that captured the exosomes containing protein markers together at $37^{\circ} \mathrm{C}$ for $1 \mathrm{~h}$, forming antibody-antigen-antibody complexes. Then, excess 


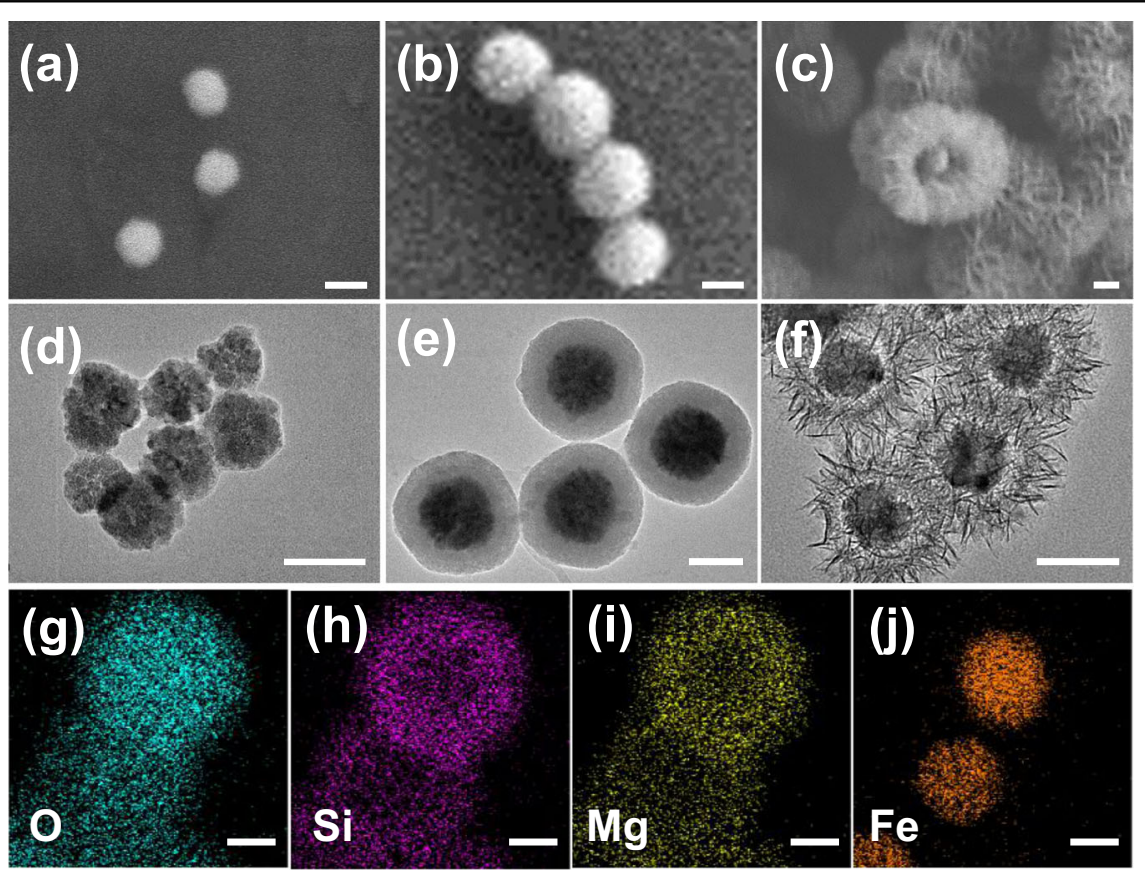

Fig. 2 Scanning electron microscopy images and transmission electron microscopy images. SEM images of a $\mathrm{Fe}_{3} \mathrm{O}_{4}$, b $\mathrm{Fe}_{3} \mathrm{O}_{4} @ \mathrm{SiO}_{2}$, and $\mathbf{c}$ $\mathrm{Fe}_{3} \mathrm{O}_{4} @ \mathrm{MgSiO}_{3}$ nanoparticles, $\mathbf{d}-\mathbf{f}$ the corresponding transmission electron microscopy image, and $\mathbf{g}-\mathbf{j}$ element mappings $(\mathrm{O}, \mathrm{Si}, \mathrm{Mg}$, and $\mathrm{Fe}$, respectively) of the $\mathrm{Fe}_{3} \mathrm{O}_{4} @ \mathrm{MgSiO}_{3}$ nanoparticles. Scale bars of $\mathbf{a}-\mathbf{f}$ represent $100 \mathrm{~nm}$, and those of $\mathbf{g}-\mathbf{j}$ represent $200 \mathrm{~nm}$.

enzyme-labeled antibodies were washed away, and $50 \mu \mathrm{L}$ substrate TMB was added to develop color by incubating for $15 \mathrm{~min}$ at $37^{\circ} \mathrm{C}$ in the dark. After $50 \mu \mathrm{L}$ stop solution was quickly dripped in the well, the content of CEA or GPC could be obtained by measuring the absorbance at $450 \mathrm{~nm}$.

\section{Clinical samples}

Two milliliters of clinical samples from patients with liver cancer $(n=10)$ and healthy donors $(n=10)$ were collected at the First Affiliated Hospital of Wenzhou Medical University with informed consent (Wenzhou, China). These blood samples were pretreated with centrifugation at $4{ }^{\circ} \mathrm{C}$ for $15 \mathrm{~min}(4000 \times g)$. After they were captured, the exosomes isolated from clinical samples were detected using the $\mathrm{Fe}_{3} \mathrm{O}_{4} @ \mathrm{MgSiO}_{3}$ nanoparticles.

\section{Statistical analysis}

All data are shown as the means \pm standard deviation. Statistical significance was assessed by a two-tailed Student's $t$-test. Values of $p<0.05$ were judged statistically significant.

\section{Results and discussion}

In a typical experiment, $\mathrm{Fe}_{3} \mathrm{O}_{4} @ \mathrm{MgSiO}_{3}$ nanoparticles were successfully synthesized via a one-step hydrothermal method. In this period, the $\mathrm{SiO}_{2}$-coated magnetic nanoparticles served as a sacrificing template to form nanoparticles with porous, burr-like shells and magnetic cores. The microstructure of the magnetic core influenced that of the fabricated nanoparticles. Thus, SEM and TEM were first chosen to observe the morphology of the magnetic colloid nanoparticles (Fig. 2a, d). This result suggested that the magnetic nanoparticles showed good monodispersity. Then, the magnetic nanoparticles were covered with silica dioxide via the modified Stöber method, forming a layer of $\mathrm{SiO}_{2}$ shell, as demonstrated in Fig. 2 b, e. It should be noted that by varying experimental conditions, nanoparticles with different shell thicknesses could be achieved. To obtain a high isolation efficiency and convenient analysis, the sizes of the chosen $\mathrm{Fe}_{3} \mathrm{O}_{4}$ cores and the sacrificed $\mathrm{Fe}_{3} \mathrm{O}_{4} @ \mathrm{SiO}_{2}$ nanoparticles were $\sim 100$ and 200 nanometers, respectively (Supplementary Fig. S1). The $\mathrm{SiO}_{2}$ shell provides silicate ions that react with the surrounding magnesium ions in the alkaline solution at high temperature to form magnesium silicate deposited on the surface of the magnetic nanoparticles. Accompanied by the consumption of $\mathrm{SiO}_{2}$, the thickness of the magnesium silicate shell increased to finally form nanostructured $\mathrm{Fe}_{3} \mathrm{O}_{4} @ \mathrm{MgSiO}_{3}$ nanoparticles. As demonstrated in Fig. 2c, f, the $\mathrm{Fe}_{3} \mathrm{O}_{4} @ \mathrm{MgSiO}_{3}$ nanoparticles had an average diameter of $250 \mathrm{~nm}$. They were composed of magnetic cores with a diameter of $100 \mathrm{~nm}$, visible cavities, spherical shells with a thickness of $10 \mathrm{~nm}$, and nanoneedles of $50 \mathrm{~nm}$ in length. Additionally, energydispersive spectroscopy elemental analysis confirmed that 
the main elements of the material were $\mathrm{O}, \mathrm{Si}, \mathrm{Mg}, \mathrm{Fe}$, suggesting the presence of magnesium silicate and iron oxide (Supplementary Fig. S2a). Furthermore, elemental mapping indicated that oxygen, silicon, and magnesium were uniformly distributed on the shells of nanoparticles and that Fe appeared only in the nucleus (Fig. $2 g-j$ and Supplementary Fig. S2b, c).

The average hydrodynamic diameters of the magnetic $\mathrm{Fe}_{3} \mathrm{O}_{4}, \mathrm{Fe}_{3} \mathrm{O}_{4} @ \mathrm{SiO}_{2}$, and $\mathrm{Fe}_{3} \mathrm{O}_{4} @ \mathrm{MgSiO}_{3}$ nanoparticles were measured to be $\sim 140,290$, and $531 \mathrm{~nm}$, indicating that the particle size gradually increased with the silicon coating and burring treatment (Fig. 3a). The polydispersity index (PDI) was 0.973 , which shows that there may have been agglomeration of $\mathrm{Fe}_{3} \mathrm{O}_{4} @ \mathrm{MgSiO}_{3}$ nanoparticles. The average diameter we measured in Fig. 3a was larger than that observed by SEM and TEM due to the presence of a hydrated layer around the particles. The XRD pattern revealed that the crystallographic structure of the core magnetic nanoparticles still existed when the magnesium silicate nanoneedle-assembled shells were formed, which agrees with the TEM results in Fig. $2 f$ (Fig. 3b). Similarly, FTIR spectra also suggested the formation of magnetic nanoparticles and $\mathrm{Fe}_{3} \mathrm{O}_{4} @ \mathrm{SiO}_{2}$ and $\mathrm{Fe}_{3} \mathrm{O}_{4} @ \mathrm{MgSiO}_{3}$ nanoparticles (Fig. 3c). The results showed that after $\mathrm{SiO}_{2}$ coating, the vibration peaks of the $\mathrm{Fe}-\mathrm{O}$ bonds shifted from 573 to $468 \mathrm{~cm}^{-1}$. Moreover, the two specific vibration peaks appeared at 796 and $954 \mathrm{~cm}^{-1}$, and an intense peak at $1107 \mathrm{~cm}^{-1}$ could be observed due to the $\mathrm{Si}-\mathrm{O}-\mathrm{Si}, \mathrm{Si}-\mathrm{OH}$, and $\mathrm{Si}-\mathrm{O}$ groups. However, the three characteristic peaks of the silica layer disappeared when the $\mathrm{Fe}_{3} \mathrm{O}_{4} @ \mathrm{MgSiO}_{3}$ nanoparticles formed, which transformed into one vibration peak at $1016 \mathrm{~cm}^{-1}$. The successful formation of the $\mathrm{MgSiO}_{3}$ nanoneedle layer could also be found in the zeta potential change, as the potential of the $\mathrm{Fe}_{3} \mathrm{O}_{4} @ \mathrm{MgSiO}_{3}$ nanoparticles increased from -57 to -20 volts (Fig. 3d). Furthermore, the $\mathrm{N}_{2}$ adsorption-desorption measurements of the $\mathrm{Fe}_{3} \mathrm{O}_{4} @ \mathrm{MgSiO}_{3}$ nanoparticles suggested that the nanoparticles had an average pore diameter of $4.07 \mathrm{~nm}$, and the BET surface area of the $\mathrm{Fe}_{3} \mathrm{O}_{4} @ \mathrm{SiO}_{2}$ increased from 18.69 to $368.2 \mathrm{~m}^{2} \mathrm{~g}^{-1}$, which was attributed to their nanoneedle and mesoporous structures. (Fig. 3e and Supplementary Fig. S3). To verify the magnetic properties of the $\mathrm{Fe}_{3} \mathrm{O}_{4} @ \mathrm{MgSiO}_{3}$ nanoparticles, the saturation magnetization value was evaluated, and it suggested good magnetic responsiveness and superparamagnetic character for instantaneous aggregation (Fig. 3f). Based on the above results, it has been proven that $\mathrm{Fe}_{3} \mathrm{O}_{4} @ \mathrm{MgSiO}_{3}$ nanoparticles can be synthesized successfully and that they possess excellent superparamagnetic properties and unique nanoneedle-assembled shell structures that give them great potential for biomolecular separation.

To achieve efficient isolation of exosomes, the $\mathrm{Fe}_{3} \mathrm{O}_{4} @ \mathrm{MgSiO}_{3}$ nanoparticles were first modified with antibodies against CD63, owing to their specific expression on the surface of exosomes. The vibration peaks at 920 and $1400 \mathrm{~cm}^{-1}$ indicated the formation of amino groups due to the treatment of APTES. In addition, two peaks corresponding to carboxylic acid groups at 1700 and $3400 \mathrm{~cm}^{-1}$ represented successful surface modification via SA treatment (Supplementary Fig. S4). The carboxyl groups of the $\mathrm{Fe}_{3} \mathrm{O}_{4} @ \mathrm{MgSiO}_{3}$ nanoparticles were further activated via EDC/NHS solution and connected to the amino groups of the CD63 antibodies. Moreover, the $\mathrm{Fe}_{3} \mathrm{O}_{4} @ \mathrm{MgSiO}_{3}$ nanoparticles were modified with fluorescein isothiocyanate (FITC)-labeled anti-CD63 antibody
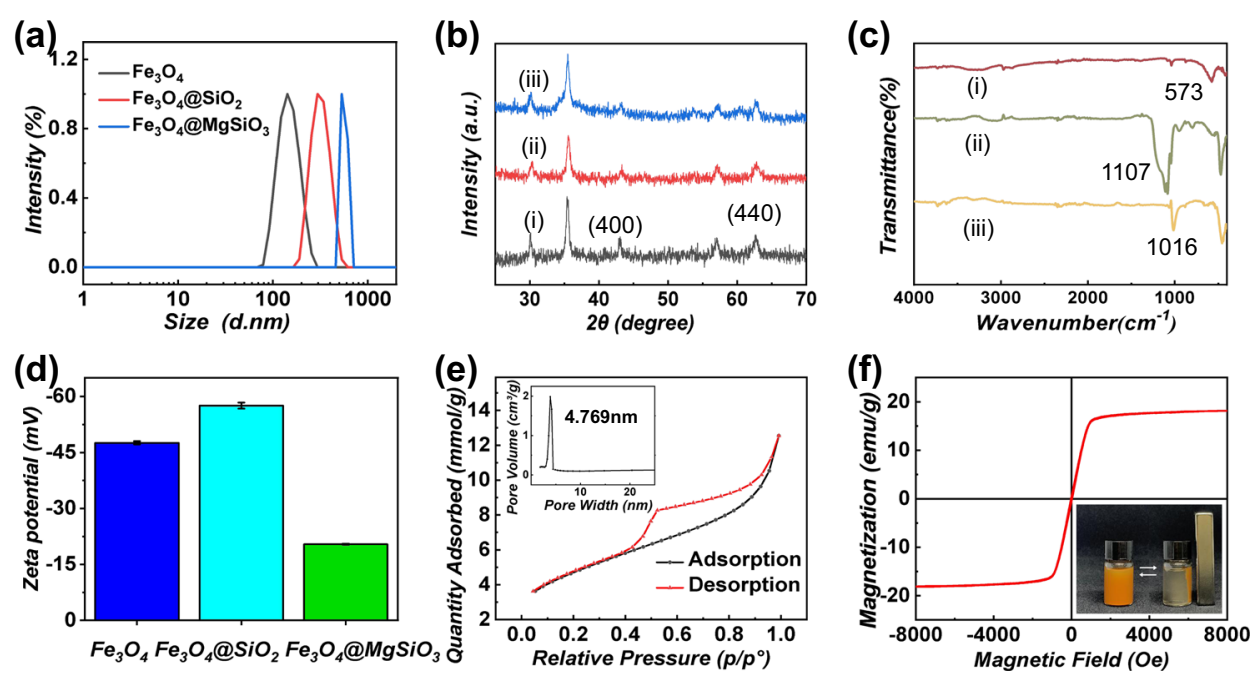

Fig. 3 Characterization of the $\mathrm{Fe}_{3} \mathrm{O}_{4}, \mathrm{Fe}_{3} \mathrm{O}_{4} @ \mathrm{SiO}_{2}$, and $\mathrm{Fe}_{3} \mathrm{O}_{4} @ \mathrm{MgSiO}_{3}$ nanoparticles. a particle size distribution, $\mathbf{b}$ XRD pattern, c FTIR spectra, and $\mathbf{d}$ zeta potential. e $\mathrm{N}_{2}$ adsorption-desorption isotherm of the $\mathrm{Fe}_{3} \mathrm{O}_{4} @ \mathrm{MgSiO}_{3}$ nanoparticles with a corresponding pore size distribution. $\mathbf{f}$ The magnetization curve of $\mathrm{Fe}_{3} \mathrm{O}_{4} @ \mathrm{MgSiO}_{3}$ nanoparticles and instantaneous magnetic responsiveness of the inserts. 


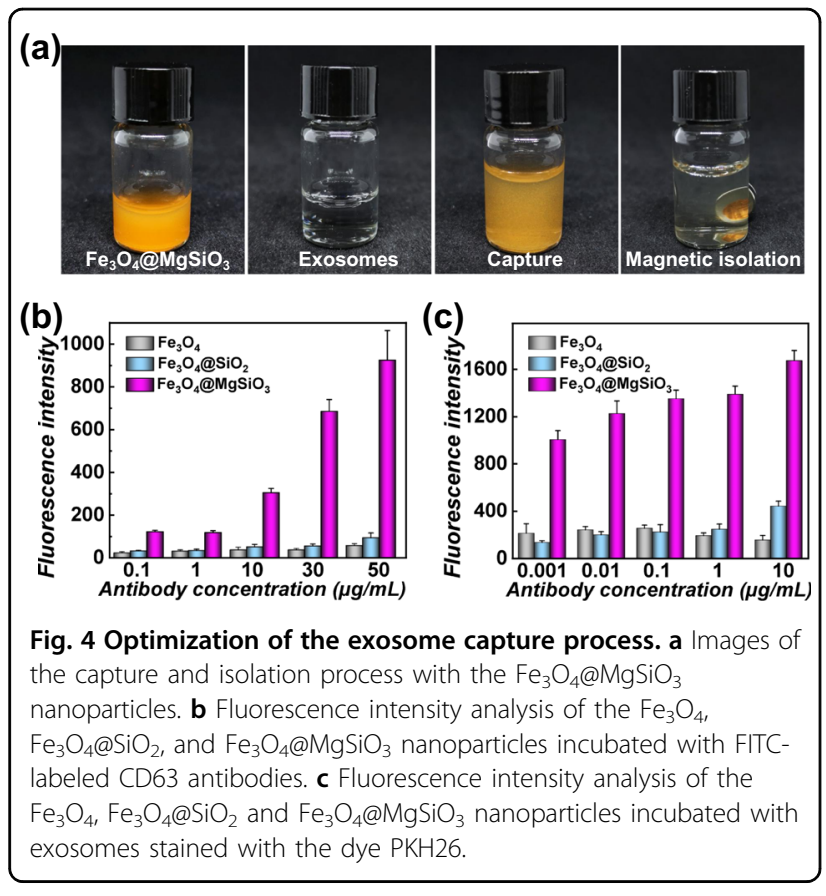

and observed by optical and fluorescence microscopy (Supplementary Fig. S5a-d). Fluorescence microscopy revealed that the modified nanoparticles had bright green fluorescence, unlike the unmodified group, suggesting that the antibody was successfully modified on the surface of the nanoparticles. After antibody modification, the $\mathrm{Fe}_{3} \mathrm{O}_{4} @ \mathrm{MgSiO}_{3}$ nanoparticles were endowed with the ability to capture and isolate exosomes efficiently, and the process is shown in Fig. 4. HepG2 cells were employed as target cells because they released high levels of exosomes. Therefore, we pre-purified exosomes from HepG2 cells by ultracentrifugation, which is considered an exosome standard. Furthermore, the ability to immobilize antibodies was compared between $\mathrm{Fe}_{3} \mathrm{O}_{4}, \mathrm{Fe}_{3} \mathrm{O}_{4} @ \mathrm{SiO}_{2}$, and $\mathrm{Fe}_{3} \mathrm{O}_{4} @ \mathrm{MgSiO}_{3}$ nanoparticles by quantitatively detecting the fluorescence intensity of FITC-labeled antibodies when modified on the above three nanoparticles (Fig. 4b). The corresponding fluorescence intensity apparently rose with increasing modified antibody concentration, and it was difficult to detect when the concentration of fluorescently labeled CD63 was lower than $1 \mu \mathrm{g} \mathrm{mL}^{-1}$. The corresponding results could also be observed by staining the exosomes with the membrane dye PKH26 (Fig. 4c). We changed the concentration range of CD63 antibody from 0.001 to $10 \mu \mathrm{g} \mathrm{mL}^{-1}$ concerning the effect of steric hindrance in the capture process, and the nanoparticles could be modified by antibodies at different concentrations. Our results confirmed that the $\mathrm{Fe}_{3} \mathrm{O}_{4} @ \mathrm{MgSiO}_{3}$ nanoparticles were significantly better than the other two nanoparticles in terms of exosome enrichment. The antibody concentrations during the reaction were further optimized to enhance the exosome capture efficiency. Different concentrations of the CD63 antibodies were immobilized on the $\mathrm{Fe}_{3} \mathrm{O}_{4} @ \mathrm{MgSiO}_{3}$ nanoparticles and then used to evaluate the effect of antibody concentration on capturing exosomes after they were blocked with BSA for $1 \mathrm{~h}$. The efficiency of capture was calculated quantitatively by detecting the number of exosomes before and after capture through the BCA method. The capture efficiency refers to the percentage of the number of captured exosomes out of the total exosomes. The capture efficiency increased gradually with increasing concentrations of CD63 antibody, and the maximum capture efficiency was achieved when the antibody concentration was increased to $10 \mu \mathrm{g} \mathrm{mL}^{-1}$ (Supplementary Fig. S6). Thus, the $\mathrm{Fe}_{3} \mathrm{O}_{4} @ \mathrm{MgSiO}_{3}$ nanoparticles were used with an optimized antibody concentration of $1 \mu \mathrm{g} \mathrm{mL}^{-1}$ to isolate exosomes effectively for subsequent downstream analyses.

As shown in Fig. 5a, the typical morphology of exosomes with saucer-like structures of $\sim 150 \mathrm{~nm}$ could be observed clearly after uranyl acetate staining. Then, the size of exosomes was measured by NanoSight, proving that the size distribution ranged from $100 \mathrm{~nm}$ to $300 \mathrm{~nm}$, and the average concentration of exosome standards was $7.4 \pm 0.38 \times 10^{9}$ particles $\mathrm{mL}^{-1}$ (Fig. 5d). Afterward, we employed the $\mathrm{Fe}_{3} \mathrm{O}_{4} @ \mathrm{MgSiO}_{3}$ nanoparticles to enrich HepG2-derived exosomes under optimized conditions. Exosomes were attached to the surfaces of the $\mathrm{Fe}_{3} \mathrm{O}_{4} @ \mathrm{MgSiO}_{3}$ nanoparticles, as observed by SEM (Fig. $5 b)$. Furthermore, the exosomes captured by the $\mathrm{Fe}_{3} \mathrm{O}_{4} @ \mathrm{MgSiO}_{3}$ nanoparticles and the exosome standards were analyzed by Western blot to detect the expression of the specific exosome marker CD63 and marker Alix and the negative marker Tommon 20 (Fig. 5c). The expression levels of CD63 in nanoparticle-captured exosomes $(\mathrm{N}$ Exos) were higher than those in original cells, which further verified that exosomes were successfully and efficiently enriched on the nanoparticles.

The protein biomarker of HepG2-derived exosomes can be used for practical disease diagnosis on account of the excellent exosome capture platform. Here, glypican-3 (GPC-3) protein and carcinoembryonic antigen (CEA) were selected as biomarkers for liver cancer diagnosis because they are overexpressed in most hepatoma cells and are located in plasma membranes. The protein biomarkers of exosomes were detected by an HRP-assisted immunosorbent assay, and the relationship between the absorbance at $450 \mathrm{~nm}$ and the concentration of GPC-3 or CEA was detected as $y=0.0458 x+0.0724$ or $y=0.0011 x+0.167$. As Fig. $5 \mathrm{e}, \mathrm{f}$ shows, along with the increased concentration of HepG2-derived exosomes captured on our nanoparticles, the measured absorbance increased. These results indicated that different concentrations of exosomes captured on the $\mathrm{Fe}_{3} \mathrm{O}_{4} @ \mathrm{MgSiO}_{3}$ nanoparticles could be quantitatively analyzed by detecting the protein levels of these biomarkers. 
(a)

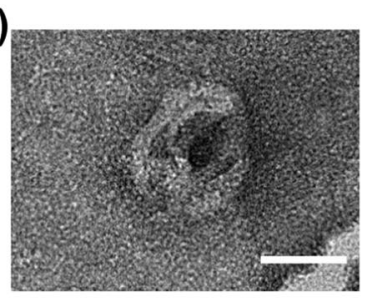

(d)

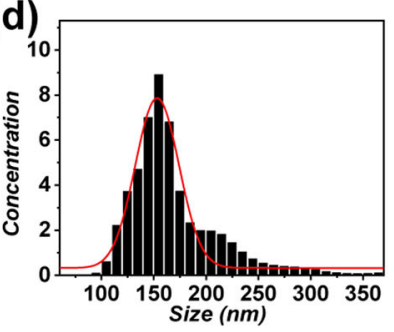

(b)

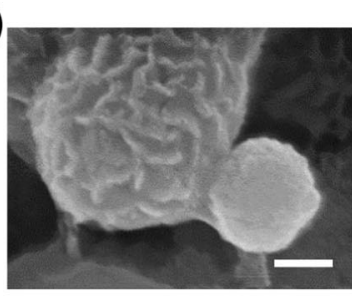

(e)

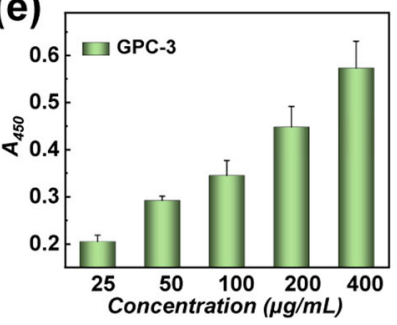

(c)

ALIX

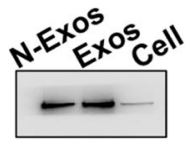

CD63

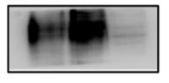

TOMM20

(f)

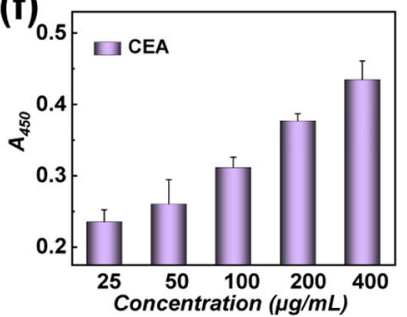

Fig. 5 Characterization and detection of captured exosomes. a TEM image of tumor-derived exosome standards; the scale bar represents $50 \mathrm{~nm}$. b SEM image of tumor-derived exosome capture onto the surface of $\mathrm{Fe}_{3} \mathrm{O}_{4} @ \mathrm{MgSiO}_{3}$ nanoparticles; the scale bar is $100 \mathrm{~nm}$. c Western blotting analysis of the proteins $\mathrm{CD63}$, Alix, and Tommon 20 in the samples of exosome-captured $\mathrm{Fe}_{3} \mathrm{O}_{4} @ \mathrm{MgSiO} \mathrm{S}_{3}$ nanoparticles and exosome standards with cell samples as the negative control. $\mathbf{d}$ The size distribution and the average concentration $\left(\times 10^{9}\right.$ particles $\left.\mathrm{mL}^{-1}\right)$ of exosome standards were measured by NanoSight. e, $\mathbf{f}$ The relationship between different concentrations of tumor-derived exosomes captured on nanoparticles and the protein levels of GPC-3 and CEA.
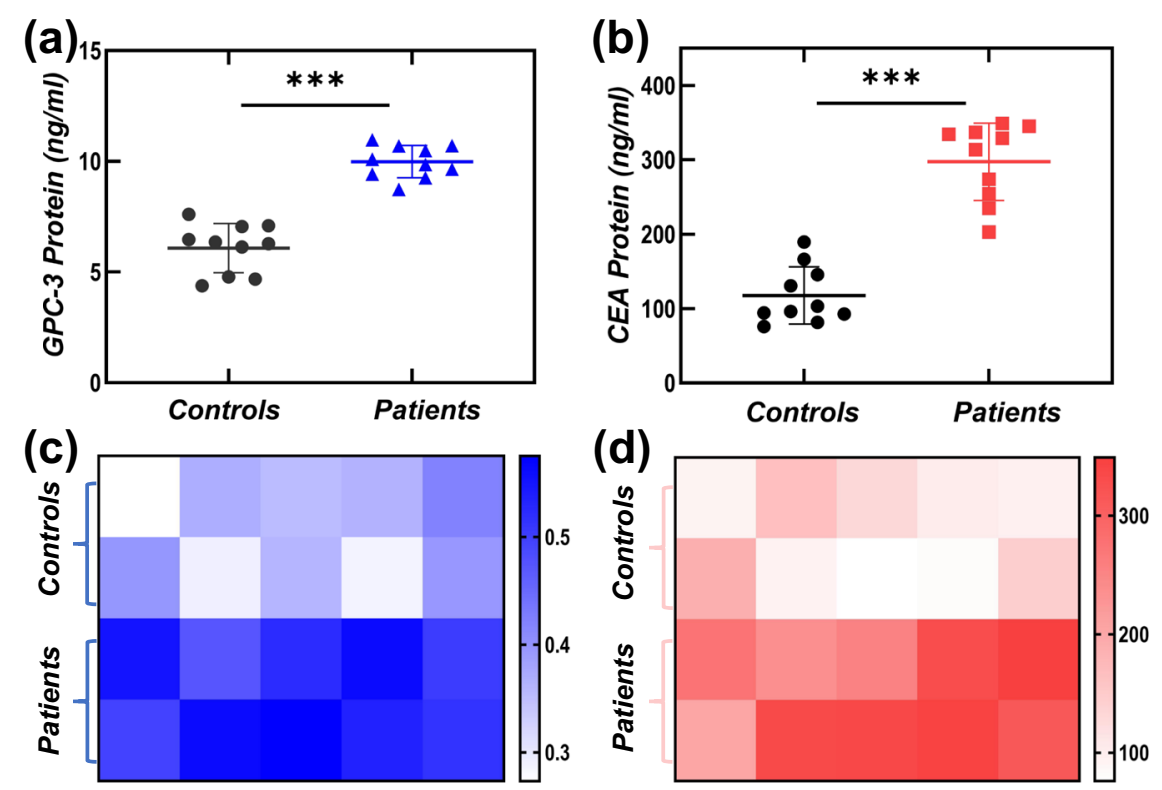

Fig. 6 Quantification of the exosomal levels. Quantification of the exosomal levels of GPC-3 (a) and CEA (b) protein in captured exosomes from the clinical samples of patients with liver cancer $(n=10)$ and healthy controls $(n=10)$. Heatmaps of GPC-3 (c) and CEA (d) protein expression levels between the patient and control groups.

To further evaluate the potential diagnostic properties of $\mathrm{Fe}_{3} \mathrm{O}_{4} @ \mathrm{MgSiO}_{3}$ nanoparticles in practice, clinical samples of ten patients with liver cancer and ten healthy donors were collected for exosome capture and detection. We compared the total amount of proteins in the serum samples using the $\mathrm{BCA}$ protein assay, but there was no significant difference between the healthy controls and the samples from the cancer patients (Supplementary Fig. S7). After the capture process, the expression levels of the two biomarker proteins in captured exosomes were 
determined. As shown in Fig. 6a, the protein level of GPC3 in patient-derived exosomes was higher than that in healthy controls. In addition, exosomal CEA could distinguish cancer patients from healthy subjects (Fig. 6b). The protein expression levels were further counted and used to construct heatmaps (Fig. 6c, d). The corresponding absorbance data of the samples are shown in Supplementary Fig. S8. These results proved that $\mathrm{Fe}_{3} \mathrm{O}_{4} @ \mathrm{MgSiO}_{3}$ nanoparticles could be used for the high enrichment and detection of exosomes derived from the plasma of clinical samples, indicating excellent application prospects for cancer diagnosis.

\section{Conclusion}

In summary, we have successfully synthesized bioinspired nanoparticles structured as nanoneedle-assembled shells and magnetic cores for isolating and detecting exosomes. Based on their fascinating physicochemical properties and excellent surface structures, the prepared multifunctional $\mathrm{Fe}_{3} \mathrm{O}_{4} @ \mathrm{MgSiO}_{3}$ nanoparticles could be modified with more probes to capture exosomes and achieve a higher yield. They could also be considered excellent nanoplatforms for efficient enrichment and detection of exosomes. Because the magnetic iron oxide cores are responsive, the exosomecombined nanoparticles can move controllably under an external magnetic field, which is beneficial for separating exosomes for detection. Furthermore, the differences in exosome analysis of clinical samples between healthy individuals and people with liver cancer can be well demonstrated. Therefore, the designed hierarchical nanoparticles have promising potential as biomarkers for early diagnosis and oncotherapy.

\section{Acknowledgements}

This work was supported by the National Key Research and Development Program of China (2020YFA0908200), the National Natural Science Foundation of China (52073060 and 61927805), and the Natural Science Foundation of Jiangsu (BE2018707).

\section{Author details}

'Department of Clinical Laboratory, Institute of Translational Medicine, The Affiliated Drum Tower Hospital of Nanjing University Medical School, Nanjing, China. ${ }^{2}$ School of Basic Medical Sciences, Henan University of Science and Technology, Luoyang, China. ${ }^{3}$ Department of Pharmacy, Jiangsu Provincial Hospital of Traditional Chinese Medicine, Nanjing, China. ${ }^{4}$ State Key Laboratory of Bioelectronics, School of Biological Science and Medical Engineering, Southeast University, Nanjing, China

\section{Author contributions}

Y.Z. conceived the idea and designed the experiment; W.Y. conducted experiments and data analysis; W.Y, Y.Y., X.S., D.Z., G.L., and Y.Z. wrote the manuscript. W.Y. and Y.Y. contributed equally to this work.

\section{Competing interests}

The authors declare no competing interests.

\section{Publisher's note}

Springer Nature remains neutral with regard to jurisdictional claims in published maps and institutional affiliations.
Supplementary information The online version contains supplementary material available at https://doi.org/10.1038/s41427-021-00346-4.

Received: 26 May 2021 Revised: 30 October 2021 Accepted: 9 November 2021.

Published online: 17 December 2021

References

1. Liebler, D. C. et al. Reassessment of exosome composition. Cell 177, 428-445 (2019).

2. Jeppesen, D. K. et al. Application of exosomes as liquid biopsy in clinical diagnosis. Signal. Transduct. Tar. 5, 1-14 (2020).

3. Kalluri, R. \& LeBleu, V. S. The biology, function, and biomedical applications of exosomes. Science 367, 6478 (2020).

4. Chen, G. et al. Exosomal PD-L1 contributes to immunosuppression and is associated with anti-PD-1 response. Nature 560, 382-386 (2018).

5. Yu, Y., Wang, Qiao, Wang, C. \& Shang, L. Living materials for regenerative medicine. Eng. Regen. 2, 96-104 (2021).

6. Wang, J. et al. Chemically edited exosomes with dual ligand purified by microfluidic device for active targeted drug delivery to tumor cells. ACS Appl. Mater. Interfaces 9, 27441-27452 (2017).

7. Wang, H., Lu, Z. \& Zhao, X. Tumorigenesis, diagnosis, and therapeutic potential of exosomes in liver cancer. J. Hematol. Oncol. 12, 1-21 (2019).

8. Di, H. et al. Nanozyme-assisted sensitive profiling of exosomal proteins for rapid cancer diagnosis. Theranostics 10, 9303 (2020).

9. Wang, W., Luo, J. \& Wang, S. Recent progress in isolation and detection of extracellular vesicles for cancer diagnostics. Adv. Health Mater. 7, 1800484 (2018).

10. Kim, S. M. et al. Cancer-derived exosomes as a delivery platform of CRISPR/ Cas9 confer cancer cell tropism-dependent targeting. J. Control. Release 26, 8-16 (2017).

11. Zhao, S., Yu, X., Qian, Y., Chen, W. \& Shen, J. Multifunctional magnetic iron oxide nanoparticles: An advanced platform for cancer theranostics. Theranostics 10, 6278 (2020).

12. $\mathrm{Hu}$, J. et al. Cell membrane-inspired polymeric vesicles for combined photothermal and photodynamic prostate cancer therapy. ACS Appl. Mater. Interfaces 12, 42511-42520 (2020).

13. Li, J., Cantu, C. P., Wang, Z. \& Zhang, Y. S. Improving bioprinted volumetric tumor microenvironments in vitro. Trends Cancer 6, 745-756 (2020).

14. Han, S. et al. Isolation and analysis of extracellular vesicles in a Morpho butterfly wing-integrated microvortex biochip. Biosens. Bioelectron. 154, 112073 (2020).

15. Song, Y., Fan, J. B., Li, X., Liang, X. \& Wang, S. pH-regulated heterostructure porous particles enable similarly sized protein separation. Adv. Mater. 31, 1900391 (2019)

16. Tian, Y. et al. Quality and efficiency assessment of six extracellular vesicle isolation methods by nano-flow cytometry. J. Extracell. Vesicles 9, 1697028 (2020).

17. $\mathrm{Qi}, \mathrm{H}$. et al. Blood exosomes endowed with magnetic and targeting properties for cancer therapy. ACS Nano 10, 3323-3333 (2016).

18. Xu, H., Liao, C., Zuo, P., Liu, Z. \& Ye, B. C. E. Magnetic-based microfluidic device for on-chip isolation and detection of tumor-derived exosomes. Anal. Chem. 90, 13451-13458 (2018).

19. $\mathrm{Ma}$, X. et al. P-glycoprotein antibody decorated porous hydrogel particles for capture and release of drug-resistant tumor cells. Adv. Health Mater. 8, 1900136 (2019).

20. $\mathrm{Ma}$, Q. et al. Cell-inspired all-aqueous microfluidics: from intracellular liquidliquid phase separation toward advanced biomaterials. Adv. Sci. 7, 1903359 (2020).

21. Wan, Y. et al. Rapid magnetic isolation of extracellular vesicles via lipid-based nanoprobes. Nat. Biomed. Eng. 1, 1-11 (2017).

22. Liao, Z., Lei, Dong \& Wang, C. Modulating, instead of suppressing, foreign body responses for biomaterials design. Eng. Regen. 2, 91-95 (2021).

23. Zhu, S. et al. Rapid multilevel compartmentalization of stable all-aqueous blastosomes by interfacial aqueous-phase separation. ACS nano $\mathbf{1 4}$ 11215-11224 (2020).

24. Liu, L. et al. A versatile dynamic mussel-inspired biointerface: from specific cell behavior modulation to selective cell isolation. Angew. Chem. 130, 8004-8008 (2018). 
25. Wang, L., Liu, H., Zhang, F., Li, G. \& Wang, S. Smart thin hydrogel coatings harnessing hydrophobicity and topography to capture and release cancer cells. Small 12, 4697-4701 (2016).

26. Zhang, X., Wang, Y., Chi, J. \& Zhao, Y. Smart microneedles for therapy and diagnosis. Research 2020, 7462915 (2020).

27. Yan, $Y$. et al. Euryale ferox seed-inspired superlubricated nanoparticles for treatment of osteoarthritis. Adv. Funct. Mater. 29, 1807559 (2019).

28. Li, H. et al. Bioinspired micropatterned superhydrophilic Au-areoles for surfaceenhanced Raman scattering (SERS) trace detection. Adv. Funct. Mater. 28, 1800448 (2018)

29. Liu, Z. et al. Multifunctional nanohybrid based on porous silicon nanoparticles, gold nanoparticles, and acetalated dextran for liver regeneration and acute liver failure theranostics. Adv. Mater. 30, 1703393 (2018).

30. Tang, H., Zhao, X. \& Jiang, X. Synthetic multi-layer nanoparticles for CRISPRCas9 genome editing. Adv. Drug Deliv. Rev. 168, 55-78 (2020).

31. Liu, Y. et al. Bioinspired structural color particles with multi-layer graphene oxide encapsulated nanoparticle components. Bioact. Mater. 5, 917-923 (2020).

32. Hou, Jue, Li, M. \& Song, Y. Recent advances in colloidal photonic crystal sensors: Materials, structures and analysis methods. Nano Today 22, 132-144 (2018).

33. Maus, A., Strait, L. \& Zhu, D. Nanoparticles as delivery vehicles for antiviral therapeutic drugs. Eng. Regen. 2, 31-46 (2021)
34. Nie, M. et al. Bio-inspired adhesive porous particles with human MSCs encapsulation for systemic lupus erythematosus treatment. Bioact. Mater. 6, 84-90 (2020).

35. Zhang, $\mathrm{H}$. et al. Fabrication of a multifunctional Nano-in-micro drug delivery platform by microfluidic templated encapsulation of porous silicon in polymer matrix. Adv. Mater. 26, 4497-4503 (2014).

36. Shin, M. K. et al. Synthesis of $\mathrm{Fe}_{3} \mathrm{O}_{4} @$ nickel-silicate core-shell nanoparticles for His-tagged enzyme immobilizing agents. Nanotechnology 27, 495705 (2016).

37. Fang, Q., Xuan, S., Jiang, W. \& Gong, X. Yolk-like micro/nanoparticles with superparamagnetic iron oxide cores and hierarchical nickel silicate shells. Adv. Funct. Mater. 21, 1902-1909 (2011).

38. Yang, J. et al. Biologically modified nanoparticles as theranostic bionanomaterials. Prog. Mater. Sci. 118, 100768 (2020).

39. Yu, Q. et al. Copper silicate hollow microspheres-incorporated scaffolds for chemo-photothermal therapy of melanoma and tissue healing. ACS Nano 12, 2695-2707 (2018).

40. Zhang, P. et al. Ultrasensitive detection of circulating exosomes with a 3Dnanopatterned microfluidic chip. Nat. Biomed. Eng. 3, 438-451 (2019).

41. Wang, Y. \& Gu, H. Core-shell-type magnetic mesoporous silica nanocomposites for bioimaging and therapeutic agent delivery. Adv. Mater. 27, 576-585 (2015). 